\title{
PLANIFICACIÓN DEL ENTRENAMIENTO EN JUEGOS SURAMERICANOS MEDELLÍN 2010 MODELOS, DURACIÓN Y CRITERIOS
}

\author{
PLANNING TRAINING IN SOUTH AMERICAN GAMES \\ MEDELLIN 2010 \\ MODELS, DURATION AND CRITERIA
}

\author{
Santiago Ramos ${ }^{1}$, Carlos Federico Ayala², Héctor Haney Aguirre 3
}

\begin{abstract}
${ }^{1}$ Magister Metodología Entrenamiento Deportivo ISCF La Habana, Cuba. Grupo de Investigación Cumanday Actividad Física y Deporte. Departamento Acción Física Humana. Universidad de Caldas, Manizales-Caldas, Colombia. Email: santiago.ramos@ucaldas.edu.co 2 Magister en Educación. Doctorando en Ciencias de la motricidad UNESP. Grupo de Investigación Cumanday Actividad Física y Deporte. Departamento Acción Física Humana. Universidad de Caldas, Manizales-Caldas, Colombia. e-mail federico.ayala@ucaldas.edu.co ${ }^{3}$ Lic. Educación Física. Estudiante Psicología. Grupo de investigación Cumanday Actividad Física y Deporte. Docente programa de Educación Física y Deportes. Universidad del Quindío. Armenia-Quindío, Colombia. e-mail. haney34@hotmail.comCorrespondencia:dirección postal calle 67 nro. 25-91 piso 3, Manizales, Caldas, Colombia.
\end{abstract}

Rev. U.D.C.A Act. E Div. Cient. 15 (Supl. Olimpismo): 67 - 76, 2012

\section{RESUMEN}

Con el objetivo de caracterizarlos modelos, las clases de ciclos utilizados, la duración y los criterios de planificación del entrenamiento deportivo entre los entrenadores participantes de los Juegos Suramericanos Medellín-2010, se realizó un estudio cuantitativo con alcance descriptivo y corte transversal. Participaron en el estudio 93 entrenadores, provenientes de 13 países, en 28 deportes, con edad media de $46,1 \pm 10,2$ años seleccionados intencionalmente. El instrumento fue una encuesta elaborada por el grupo investigador, validada por juicio de expertos, sobre las características de planificación. Los entrenadores fueron encuestados en los sitios de competencia o descanso, previa firma del consentimiento informado. Se encontró que la experiencia media como entrenadores fue 20,5 $\pm 9,8$ años. Los deportes, se agruparon en Resistencia, en Fuerza-velocidad, en Pelota, en Combate y en Precisión y Arte Competitivo. Se encontró que la planificación se orientó, principalmente, a largo y medio plazo. Los modelos de planificación más utilizados fueron tradicional, ciclos, bloques e individual. Sigue predominando el modelo tradicional de periodización; se registraron diferencias en función del grupo de deporte y de la clasificación final de países por medallas de oro. Finalmente, de acuerdo a los resultados, se recomienda instaurar procesos de ca- pacitación y de formación en modelos contemporáneos de planificación deportiva.

Palabras clave: Entrenamiento deportivo, planificación del entrenamiento, periodización, modelos de planificación.

\section{SUMMARY}

In order to characterize the used models, the classes of cycles, the duration, and the planning criteria of sports training by the participating coaches of the South American Games Medellin-2010, a quantitative study with cross sectional range was conducted. In the research participated 93 coaches from 13 countries in 28 sports, with a mean age of $46.1 \pm 10.2$ years, selected intentionally. The instrument was a survey, conducted by the research group, validated by experts, on the characteristics of planning. Coaches were surveyed at sites of competition or at rest, after signing informed consent. It was found that the average experience as coaches was $20.5 \pm 9.8$ years. Sports are grouped in Resistance, Strength, Speed, Ball, Combat and Accuracy and Competitive Art. It was found that planning was geared mainly at medium and long term. The planning models most used were the traditional, cycles, blocks and the individual one. The traditional model of periodization continues to 
dominate. Differences were found according to the group of sports and the final ranking of countries by gold medals. Finally, according to the results, it is recommended to establish training and formation processes in contemporary models of sports planning.

Key words: Sports training, planning, training, periodization, planning models.

\section{INTRODUCCIÓN}

Es posible caracterizar la evolución histórica de la planificación del entrenamiento deportivo en tres etapas: la primera, desde los orígenes más antiguos hasta 1950, donde básicamente cuenta la experiencia individual y se hacen unos primeros intentos de sistematización; la segunda, entre 1950 y 1970, con sistemas que tienen bases científicas con gran aporte, especialmente, del bloque ex socialista y, la tercera, a partir de 1980, donde se superan las teorías clásicas (Martins et al. 1999, citado por Dantas et al. 2010). La "periodización del entrenamiento" tradicional fue propuesta por Matveyev (1977), cuando el entrenamiento no tenía una gran fundamentación científica. Desde entonces, el deporte y las ciencias del deporte han experimentado cambios profundos (Issurin, 2010), mientras que la periodización tradicional se ha mantenido más o menos al mismo nivel (Navarro et al. 2010).

La dinámica del deporte internacional, en especial, el marcado aumento del número de competencias y el tiempo durante el cual se realizan, ha generado cambios en la metodología y en la planificación del entrenamiento, entre ellos: 1) La modificación radical de las condiciones económicas, sociales, organizativas y profesionales del deportista de alto rendimiento; 2) Las circunstancias en que se encuentra el deporte, lo que le obliga a apoyarse en otras ciencias; 3) Conceptualización de términos clásicos de la metodología del entrenamiento que han sido reestructurados y 4) Nuevos y alternativos conceptos que no rechazan la teoría clásica de Matveyev (1977), sino que la complementan (González et al. 2007).

Según Vasconcelos (2005), “desarrollar las capacidades motoras constituye la condición física necesaria para buscar un rendimiento deportivo elevado". Por otro lado, las preparaciones técnica y táctica están unidas, ya que las primeras son la base de las acciones tácticas, pues estas son diversificadas, y acordes a la situación. La preparación intelectual busca mejorar los conocimientos del deportista, para que los aplique creativamente en la preparación y en la competición. Finalmente, la preparación psicológica busca potencializar la voluntad y la motivación del deportista frente a las exigencias de la competencia y el entrenamiento, pues "la preparación psicológica busca acelerar y facilitar el proceso de integración y control sobre los diferentes sistemas y procesos envueltos en la prestación deportiva" (Vasconcelos, 2005). Según el mismo autor, la planificación es "anticipar, prever una secuencia lógica y coherente del desarrollo de las tareas que nos llevan a alcanzar objetivos previamente definidos", no es algo nuevo, pues desde épocas remotas se han venido estructurando los entrenamientos para toda clase de actividades, en las cuales, el ser humano podía participar.

Weineck (2005) consideró que la periodización del entrenamiento deportivo se centra en la rotación, de lo que denomina ciclos repetidos y su manifestación se da en el periodo preparatorio, buscando el desarrollo de la forma deportiva; en tanto que el de competencias apunta al progreso en la misma forma deportiva y, finalmente, el de transición, persigue la descarga; así mismo, en dicho proceso, se encuentran unas subdivisiones que permiten organizar en mejores condiciones el proceso de entrenamiento.

Los objetivos competitivos que van dando el nivel del deportista, el calendario de competencias y la duración de la forma deportiva son los criterios fundamentales que determinan la planificación del entrenamiento.

La clasificación de los deportes ha tenido distintas taxonomías; la utilizada en este trabajo es de acuerdo a afinidades metodológicas y biológicas: deportes de resistencia, de fuerza-velocidad, de combate, con pelota y de precisión y arte competitivo (PAC ahora en adelante) (Acevedo, 2010).

La primera revisión en inglés de la teoría de la periodización de Matveyev por Frank Dick, en Gran Bretaña, 1975, causó el mayor cambio en el paradigma de la planificación del entrenamiento de los atletas, tanto que para el cambio al siglo XXI, la periodización formaba los fundamentos de las más modernas teorías y prácticas y las bases de todo entrenamiento atlético serio. Para las décadas entre 1950 y 1970, el modelo de Matveyev fue aplicado a la mayoría de modalidades y se estableció como modelo científico, reconociéndose tales aportes y fundamentos de la periodización, como la perspectiva o teoría Tradicional (Issurin, 2012). Durante este tiempo, la teoría tradicional se aceptó como base universal del entrenamiento; no obstante, y según Issurin (2012), para la década de 1980, aparecieron las críticas y las tendencias de modificación, que contrastaban con la perspectiva tradicional. En este sentido, Issurin (2012) señala que la incapacidad de proporcionar una participación exitosa en competiciones ubicadas en un período largo de tiempo constituía una de las principales debilidades del modelo de Matveyev, además de otras limitaciones, como: a) Restricciones en el desarrollo paralelo de capacidades motrices y técnicas y, b) 
Limitaciones impuestas por períodos excesivamente prolongados en la preparación básica y específica.

Reconocer tales cambios que se enmarcan en transformaciones contextuales políticas, sociales, económicas y científicas, se constituye en una de las tareas en las que se fija la tendencia de la preparación del entrenamiento deportivo. Para el contexto suramericano aún no se tiene apropiado un cuerpo teórico y metodológico que señale una tendencia, como los ejercidos por el modelo tradicional y las modificaciones de las perspectivas contemporáneas.

De este modo, el propósito de la presente investigación fue caracterizar los modelos, los procesos, la duración y los criterios de planificación del entrenamiento deportivo utilizados por los entrenadores participantes en los Juegos Suramericanos Medellín-2010.

\section{MATERIALES Y MÉTODOS}

El estudio fue de tipo cuantitativo, de alcance descriptivo y corte transversal, como lo definen Hernández et al. (2010). Contestaron la encuesta 93 técnicos deportivos participantes en los Juegos Suramericanos Medellín 2010 (JSA), seleccionados por conveniencia. No fue posible obtener información de la organización del evento, acerca del total de entrenadores participantes en los Juegos. Los datos fueron recolectados entre el 23 y el 27 de marzo de 2010, durante los JSA. Se contactaron a los técnicos en los diferentes escenarios deportivos y lugares de descanso, donde se les informó sobre el objetivo de la investigación. Se les entregó el consentimiento informado, en el cual, se informaba que la participación sería voluntaria, se les garantizaba el anonimato y la garantía que la información recogida tendría únicamente uso académico y, finalmente, se les aplicó la encuesta, teniendo presente la Resolución 8430/1993, Art. 11, dado que la aplicación del instrumento no implicaba ningún riesgo para los participantes en el estudio. Fueron entrenados cuatro estudiantes y siete licenciados en educación física, para la aplicación del instrumento.

La encuesta contenía 45 preguntas elaboradas por el equipo investigador, referentes a diferentes aspectos de la metodología del entrenamiento deportivo que, para el presente artículo, fueron seleccionados los atinentes a modelos de planificación empleados y tiempo y criterios de planificación. Después de someterla a criterio de expertos, se le hizo la prueba piloto con 12 entrenadores deportivos del Departamento de Caldas, para su posterior ajuste. La encuesta, en español, tenía una duración aproximada de 25 minutos y fue aplicada por los evaluadores o dejada en poder de algunos entrenadores para que la contestaran y la devolvieran.
Se elaboró una base de datos en el programa Excel 2007, donde se clasificaron las diversas preguntas para hacer análisis de frecuencias, de porcentajes, de promedios y de desviación estándar.

\section{RESULTADOS Y DISCUSIÓN}

Características socio-demográficas de la muestra evaluada: Los 93 entrenadores provenían de 13 de los 15 países participantes en los Juegos (86,6\%), en 28 de las modalidades programadas (75,7\%). La edad media fue de 46,1 $\pm 10,2$ (entre 24 y 79 años). Por sexos, hubo 84 hombres (90,3\%) y siete mujeres $(9,7 \%)$, provenientes, cuatro de ellas, de la gimnasia rítmica con elementos.

La formación académica muestra un $94 \%$ de los entrenadores con formación post-secundaria y un $85,4 \%$ a nivel profesional universitario o de postgrado. Además, existen diferencias entre los países, según la clasificación final por medallas de oro, pues los tres primeros tuvieron un $88 \%$ de sus entrenadores entre pregrado $(37 \%)$ y postgrado $(51 \%)$; los clasificados entre $4^{\circ}$ y $7^{\circ}$ tuvieron un $83,2 \%$ de sus entrenadores en esas mismas categorías $(52,7 \%$ y $30,5 \%$, respectivamente). Los últimos países en el medallero, por su parte, tuvieron un $76,8 \%$, en dichas categorías, con un $46,1 \%$, con pregrado y $30,7 \%$, con postgrado.

En función del grupo de deporte (Tabla 1), se encontró que el grupo de fuerza-velocidad tiene más entrenadores con formación de postgrado, mientras que los grupos de pelota y PAC son los más homogéneos en la formación de sus técnicos.

En cuanto al nivel de participación como entrenadores, la mayoría ha alcanzado el nivel de Juegos Panamericanos. Por otra parte, destacan los grupos de deportes de resistencia y de fuerza-velocidad, en los cuales, casi tres de cada cinco han llevado delegaciones a campeonatos mundiales o Juegos Olímpicos. Por el contrario, la mayoría del grupo de deportes de pelota enuncia el suramericano, como su máximo nivel alcanzado. Por resultados finales, entre los primeros países clasificados, el $60 \%$ de los entrenadores ha llevado delegaciones a campeonatos mundiales o Juegos Olímpicos, frente a un $41 \%$, de los países clasificados del $4^{\circ}$ al $7^{\circ}$ y un $21 \%$, del resto de países participantes. En cuanto a la productividad intelectual o académica, solamente el $41 \%$ ha participado alguna vez en investigación y un 33\%, ha publicado algún artículo o libro relacionado con el deporte.

Con base en los resultados anteriores, se puede afirmar que es conveniente que los entrenadores deportivos posean una formación académica, por lo menos de tercer ciclo 
Tabla 1. Características socio-demográficas de los entrenadores suramericanos por grupos de deportes.

\begin{tabular}{|c|c|c|c|c|c|c|c|c|c|c|}
\hline \multirow{2}{*}{ Variable } & \multicolumn{2}{|c|}{ Resist } & \multicolumn{2}{|c|}{ Fza-vel } & \multicolumn{2}{|c|}{ Pelota } & \multicolumn{2}{|c|}{ Combate } & \multicolumn{2}{|c|}{ PAC } \\
\hline & $\mathrm{n}$ & $\%$ & $\mathrm{~N}$ & $\%$ & $\mathrm{n}$ & $\%$ & $\mathrm{n}$ & $\%$ & $n$ & $\%$ \\
\hline \multicolumn{11}{|l|}{ Género } \\
\hline Masculino & 8 & 8,6 & 16 & 17,2 & 36 & 38,7 & 15 & 16,1 & 11 & 11,8 \\
\hline Femenino & 1 & 1,1 & 0 & 0,0 & 1 & 1,1 & 0 & 0,0 & 5 & 5,4 \\
\hline \multicolumn{11}{|l|}{ Edad (años) } \\
\hline Entre 24 y 41 & 2 & 2,15 & 3 & 3,23 & 12 & 12,90 & 2 & 2,15 & 5 & 5,38 \\
\hline Entre 42 y 59 & 5 & 5,38 & 10 & 10,75 & 22 & 23,66 & 10 & 10,75 & 6 & 6,45 \\
\hline Entre 60 y 79 & 2 & 2,15 & 2 & 2,15 & 2 & 2,15 & 1 & 1,08 & 1 & 1,08 \\
\hline Perdidos & 0 & 0,00 & 1 & 1,08 & 1 & 1,08 & 2 & 2,15 & 4 & 4,30 \\
\hline \multicolumn{11}{|l|}{ Escolaridad } \\
\hline Básica & 0 & 0,0 & 1 & 1,1 & 0 & 0,0 & 0 & 0,0 & 0 & 0,0 \\
\hline Técnica & 1 & 1,1 & 1 & 1,1 & 0 & 0,0 & 3 & 3,2 & 0 & 0,0 \\
\hline Tecnológica & 0 & 0,0 & 1 & 1,1 & 4 & 4,3 & 1 & 1,1 & 1 & 1,1 \\
\hline Universitaria & 4 & 4,3 & 5 & 5,4 & 18 & 19,3 & 7 & 7,5 & 7 & 7,5 \\
\hline Postgraduada & 3 & 3,2 & 7 & 7,5 & 12 & 12,9 & 3 & 3,2 & 5 & 5,4 \\
\hline Perdidos & 1 & 1,1 & 1 & 1,1 & 3 & 3,2 & 1 & 1,1 & 3 & 3,2 \\
\hline \multicolumn{11}{|c|}{ Experiencia como entrenador } \\
\hline Olímpicos & 3 & 3,2 & 7 & 7,5 & 5 & 5,4 & 2 & 2,2 & 3 & 3,2 \\
\hline Mundiales & 4 & 4,3 & 5 & 5,4 & 3 & 3,2 & 1 & 1,1 & 3 & 3,2 \\
\hline Panamericanos & 2 & 2,2 & 1 & 1,1 & 3 & 3,2 & 4 & 4,3 & 6 & 6,4 \\
\hline Centroamericano & 0 & 0,0 & 0 & 0,0 & 1 & 1,1 & 4 & 4,3 & 0 & 0,0 \\
\hline Suramericanos & 0 & 0,0 & 3 & 3,2 & 10 & 10,7 & 3 & 3,2 & 2 & 2,2 \\
\hline Bolivarianos & 0 & 0,0 & 0 & 0,0 & 2 & 2,2 & 0 & 0,0 & 0 & 0,0 \\
\hline Nacional & 0 & 0,0 & 0 & 0,0 & 6 & 6,4 & 0 & 0,0 & 1 & 1,1 \\
\hline Otro & 0 & 0,0 & 0 & 0,0 & 0 & 0,0 & 1 & 1,1 & 0 & 0,0 \\
\hline Perdidos & 0 & 0,0 & 0 & 0,0 & 7 & 7,5 & 0 & 0,0 & 1 & 1,1 \\
\hline Total & 9 & 9,7 & 16 & 17,2 & 37 & 39,8 & 15 & 16,1 & 16 & 17,2 \\
\hline
\end{tabular}

Fuente propia. Nota: Resist=deportes de resistencia, Fza-vel=deportes de fuerza-velocidad, PAC=deportes de Precisión y arte competitivo.

(postgrado en el área), pues esta tiene relación con los procesos que debería poseer un entrenador de este nivel deportivo y las exigencias del mismo; aunque Salinero \& Ruiz (2010) informan que de los entrenadores de alto rendimiento en Karate, el 83,3\% no tienen estudios superiores y tampoco toman parte en cursos o actividades de formación; son entrenadores con una amplia experiencia deportiva y competitiva, por lo que su conocimiento es del campo de la competición.
Modelos y criterios de planificación del entrenamiento utilizados: Los modelos de planificación más utilizados fueron el tradicional, ciclos, bloques, individual y el de acumulación, transformación y realización (ATR), este último, dominante en los modelos contemporáneos (Tabla 2). Una revisión sistemática realizada por Dantas et al. (2010) arrojó que los modelos de periodización tradicional, ATR y campanas estructurales presentaron un Índice de Aplicabilidad (IA) 
Tabla 2. Modelos y criterios de planificación utilizados por los entrenadores suramericanos.

\begin{tabular}{|c|c|c|c|c|c|c|c|c|c|c|c|c|}
\hline \multirow{2}{*}{ Variables } & \multicolumn{2}{|c|}{$\begin{array}{l}\text { Todos } \\
(\mathrm{n}=93)\end{array}$} & \multicolumn{2}{|c|}{$\begin{array}{l}\text { Resist } \\
(n=9)\end{array}$} & \multicolumn{2}{|c|}{$\begin{array}{l}\text { Fza-vel } \\
(n=16)\end{array}$} & \multicolumn{2}{|c|}{$\begin{array}{l}\text { Pelota } \\
(\mathrm{n}=37)\end{array}$} & \multicolumn{2}{|c|}{$\begin{array}{l}\text { Combate } \\
(n=15)\end{array}$} & \multicolumn{2}{|c|}{$\begin{array}{c}\text { PAC. } \\
(n=16)\end{array}$} \\
\hline & $\mathrm{N}$ & $\%$ & $\mathrm{n}$ & $\%$ & $\mathrm{n}$ & $\%$ & $\mathrm{n}$ & $\%$ & $\mathrm{n}$ & $\%$ & $\mathrm{n}$ & $\%$ \\
\hline \multicolumn{13}{|c|}{ Modelos de planificación utilizados* } \\
\hline Períodos & 49 & 25,1 & 3 & 12,5 & 11 & 23,4 & 18 & 25,0 & 11 & 26,8 & 6 & 30,0 \\
\hline Ciclos & 35 & 17,9 & 3 & 12,5 & 7 & 14,9 & 14 & 19,4 & 6 & 14,6 & 5 & 25,0 \\
\hline Bloques & 26 & 13,3 & 3 & 12,5 & 9 & 19,1 & 10 & 13,9 & 3 & 7,3 & 1 & 5,0 \\
\hline Individual & 26 & 13,3 & 2 & 8,3 & 4 & 8,5 & 12 & 16,7 & 4 & 9,8 & 4 & 20,0 \\
\hline ATR & 24 & 12,3 & 7 & 29,2 & 4 & 8,5 & 8 & 11,1 & 5 & 12,2 & 0 & 0,0 \\
\hline Altascargas & 7 & 3,6 & 1 & 4,2 & 3 & 6,4 & 3 & 4,2 & 0 & 0,0 & 0 & 0,0 \\
\hline Campanas & 7 & 3,6 & 1 & 4,2 & 3 & 6,4 & 0 & 0,0 & 0 & 0,0 & 0 & 0,0 \\
\hline Macros.Int & 15 & 7,7 & 2 & 8,3 & 2 & 4,3 & 5 & 6,9 & 12 & 29,3 & 3 & 15,0 \\
\hline Orga.Lóg. & 6 & 3,1 & 2 & 8,3 & 4 & 8,5 & 2 & 2,8 & 0 & 0,0 & 1 & 5,0 \\
\hline \multicolumn{13}{|c|}{ Criteriospara la planificación* } \\
\hline Calendario & 67 & 35,4 & 5 & 31,3 & 15 & 33,3 & 26 & 31,7 & 13 & 40,6 & 8 & 44,4 \\
\hline Objetivos & 66 & 34,9 & 5 & 31,3 & 15 & 33,3 & 37 & 45,1 & 12 & 37,5 & 1 & 5,6 \\
\hline Deportista & 46 & 24,3 & 6 & 37,5 & 11 & 24,4 & 14 & 17,1 & 7 & 21,9 & 8 & 44,4 \\
\hline Otros & 10 & 5,3 & 0 & 0,0 & 4 & 8,9 & 5 & 6,1 & 0 & 0,0 & 1 & 5,6 \\
\hline
\end{tabular}

Nota:Resist $=$ deportes de resistencia, Fza-vel=deportes de fuerza-velocidad, PAC=deportes de Precisión y arte competitivo, Macrosint=macrociclos integrados, Orga.Lóg=organigramas lógicos.

* Los entrenadores podían seleccionar más de una respuesta.

"Muy Bueno", y los modelos Prioritario y en Bloques, "Bueno". Por lo que estos modelos con un IA "Muy Bueno" son los de mejor aplicabilidad.

En los deportes de resistencia, se encuentra una predominancia del modelo ATR, seguido de tradicional, ciclos y bloques; el modelo tradicional sobresale en los deportes de fuerza-velocidad, seguido de los bloques y ciclos. En los deportes de pelota, nuevamente, el modelo sobresaliente es el tradicional, seguido del de ciclos y el individual; en los de combate, el tradicional es el amplio dominador ante el de ciclos. En los de PAC, el tradicional fue predominante, seguido de ciclos e individual. Se encontró que el modelo de planificación más utilizado en los JSA fue el tradicional, seguido de ciclos y bloques, respectivamente; de los modelos contemporáneos el que más se utiliza es el ATR, ampliamente sobre los macros integrados, altas cargas y campanas estructurales, respectivamente.
Una posible explicación de la predominancia del ATR entre los modelos contemporáneos, es por: (i) la sustitución de cargas regulares -modelo tradicional- por la concentración de cargas -Modelos de bloques- (Manzano, 2004), siendo este elemento "el principio más decisivo e importante en la planificación por bloques" (Issurin, 2012); (ii) la duración de cada uno de los Mesociclos dependerá del modelo de temporada y se establece de acuerdo al calendario de competencias (Manzano, 2004), es decir, los ciclos medios o mesociclos es el concepto más fundamental en el ATR. Issurin (2012) precisa que son más concentrados y más manejables en todos los programas de entrenamiento, lo cual, en la secuencia racional de los mesociclos permite la obtención de una superposición óptima de los efectos residuales del entrenamiento y como resultado un rendimiento de competición a alto nivel en todas las capacidades motoras y técnicas y, (iii) cada uno de mesociclos de la periodización del entrenamiento depende de las particularidades de cada deporte. 
Según los criterios utilizados para la planificación, en su orden general, están el calendario de competencias, seguido de los objetivos y las características del deportista. Los deportes de resistencia centran la planeación esencialmente en el deportista, seguido del calendario de competencias y los objetivos. Los entrenadores de fuerza-velocidad y de combate, se centran en el calendario de competencias y los objetivos, seguido de las características del deportista; en los deportes de pelota, la planificación se centra en los objetivos ampliamente, luego el calendario de competencias y las condiciones del deportista. Los PAC tienen como referencia el calendario y las condiciones del deportista preferiblemente.

En conclusión, encontramos que el calendario de competencias es el mayor criterio que se tiene en cuenta para la planificación del entrenamiento deportivo, seguido de cerca por los objetivos y las características y condiciones del deportista. Esto puede estar determinado por condiciones objetivas, como la contratación por corto tiempo de los entrenadores, pero según la literatura, no es la mejor opción como criterio, debiendo ser en primer lugar los objetivos de desarrollo del deportista.

Los teóricos del entrenamiento deportivo (Matveyev, 1977, 2001; Vasconcelos, 2005; Bompa, 2003) proponen la planificación a largo plazo como la tendencia actual más importante para el desarrollo global del deportista; no obstante, algunos de los entrenadores participantes en los JSA no pueden hacerlo, pues su vinculación con las selecciones nacionales es incierta y a corto plazo, lo que constituye un indudable freno a las posibilidades reales de desarrollo de los deportistas; dicha planificación a largo plazo fue la de mayor frecuencia de respuestas entre los entrenadores encuestados, seguida del mediano plazo, Olímpico y anual.

Tabla 3. Tipos de mesociclos y de microciclos utilizados por los entrenadores suramericanos.

\begin{tabular}{|c|c|c|c|c|c|c|c|c|c|c|c|c|}
\hline \multirow[t]{2}{*}{ Caract. } & \multicolumn{2}{|c|}{$\begin{array}{l}\text { Todos } \\
(\mathrm{n}=93)\end{array}$} & \multicolumn{2}{|c|}{$\begin{array}{c}\text { Resisten } \\
(\mathrm{n}=9)\end{array}$} & \multicolumn{2}{|c|}{$\begin{array}{l}\text { Fza-vel } \\
(n=16)\end{array}$} & \multicolumn{2}{|c|}{$\begin{array}{l}\text { Pelota } \\
(\mathrm{n}=37)\end{array}$} & \multicolumn{2}{|c|}{$\begin{array}{c}\text { Combate } \\
(n=15)\end{array}$} & \multicolumn{2}{|c|}{$\begin{array}{c}\text { PAC } \\
(n=16)\end{array}$} \\
\hline & $\mathrm{n}$ & $\%$ & $\mathrm{n}$ & $\%$ & $\mathrm{n}$ & $\%$ & $\mathrm{n}$ & $\%$ & $\mathrm{n}$ & $\%$ & $\mathrm{n}$ & $\%$ \\
\hline \multicolumn{13}{|c|}{ Tipos de mesociclos utilizados } \\
\hline Competi. & 52 & 18,6 & 6 & 19,4 & 8 & 19,0 & 18 & 16,5 & 12 & 18,5 & 8 & 18,6 \\
\hline Especial & 50 & 17,9 & 7 & 22,6 & 7 & 16,7 & 19 & 17,4 & 9 & 13,8 & 8 & 18,6 \\
\hline Control & 44 & 15,7 & 4 & 12,9 & 4 & 9,5 & 20 & 18,3 & 7 & 10,8 & 9 & 20,9 \\
\hline Precomp & 39 & 13,9 & 4 & 12,9 & 4 & 9,5 & 16 & 14,7 & 17 & 26,2 & 8 & 18,6 \\
\hline De base & 38 & 13,6 & 3 & 9,7 & 8 & 19,0 & 12 & 11,0 & 10 & 15,4 & 5 & 11,6 \\
\hline Gradual & 25 & 8,9 & 2 & 6,5 & 4 & 9,5 & 11 & 10,1 & 4 & 6,2 & 4 & 9,3 \\
\hline Recupera & 25 & 8,9 & 4 & 12,9 & 4 & 9,5 & 10 & 9,2 & 6 & 9,2 & 1 & 2,3 \\
\hline Otro & 6 & 2,1 & 1 & 3,2 & 2 & 4,8 & 3 & 2,8 & 0 & 0,0 & 0 & 0,0 \\
\hline Ninguno & 1 & 0,4 & 0 & 0,0 & 1 & 2,4 & 0 & 0,0 & 0 & 0,0 & 0 & 0,0 \\
\hline \multicolumn{13}{|c|}{ Tipos de microciclos utilizados } \\
\hline Choque & 57 & 18,8 & 5 & 19,2 & 11 & 18,0 & 22 & 18,8 & 9 & 14,8 & 10 & 22,2 \\
\hline Compet & 56 & 18,5 & 5 & 19,2 & 10 & 16,4 & 23 & 19,7 & 11 & 18,0 & 7 & 15,6 \\
\hline Precomp & 53 & 17,5 & 5 & 19,2 & 6 & 9,8 & 22 & 18,8 & 12 & 19,7 & 8 & 17,8 \\
\hline Regener & 52 & 17,2 & 5 & 19,2 & 8 & 13,1 & 19 & 16,2 & 12 & 19,7 & 8 & 17,8 \\
\hline Corriente & 36 & 11,9 & 2 & 7,7 & 8 & 13,1 & 13 & 11,1 & 9 & 14,8 & 4 & 8,9 \\
\hline Gradual & 36 & 11,9 & 2 & 7,7 & 15 & 24,6 & 15 & 12,8 & 7 & 11,5 & 4 & 8,9 \\
\hline Otro & 13 & 4,3 & 2 & 7,7 & 3 & 4,9 & 3 & 2,6 & 1 & 1,6 & 4 & 8,9 \\
\hline
\end{tabular}

Nota: Resist=deportes de resistencia, Fza-vel=deportes de fuerza-velocidad, PAC=deportes de Precisión y arte competitivo, Precomp $=$ precompetitivo, competi $=$ competitivo, regener $=$ regenerativo. 
Mesociclos y microciclos utilizados por los entrenadores suramericanos: Entendiendo que un mesociclo es un periodo de preparación entre dos y seis semanas (microciclos) y, en algunos casos, algo más (Harre, 1983;Vasconcelos, 2005;Platonov, 2001; Navarro, 2003; Weineck 2005);según la tabla 3, se encontró que los mesociclos más utilizados son los competitivos y especiales, que se orientan en el periodo competitivo en la planificación tradicional, lo que determina que estos procesos se centran en la competencia. Se resaltan los mesociclos de control, mientras los mesociclos precompetitivos y de base siguen con un porcentaje bajo, lo que, posiblemente, se debe a que los entrenadores desarrollan planes para deportistas ya formados y de alto nivel. En pocos casos, se observa la importancia de la recuperación y la gradualidad de la planificación por mesociclos.

En los deportes de resistencia la planificación se centra en lo competitivo y lo específico del proceso destacándose los mesociclos de control, recuperación y precompetitivo. En los deportes de fuerza-velocidad, se recoge la relación que los entrenadores le dan entre la competencia y la preparación de base, aunque en sumatoria, se sigue privilegiando la competencia y la preparación específica. En deportes de pelota, le dan relevancia a los mesociclos de control. Los deportes de combate centran su interés en los mesociclos de precompetencia, para luego orientarlos hacia la competencia. Los deportes de PAC le dan bastante relevancia a los mesociclos de control, lo cual, es característico de este tipo de deportes, pues es la base del periodo competitivo, como lo evidencian los resultados de la tabla 3.

Según Issurin (2012), el concepto de periodización en bloques propone tres tipos de mesociclos: Acumulación, Transformación y Realización -ATR-), que difieren considerablemente de la teoría del entrenamiento tradicional, lo que explica lo dicho anteriormente sobre la influencia de este modelo en el ámbito suramericano. Los entrenadores suramericanos priorizaron los mesociclos competitivos y especiales, seguidos de cerca por los de control; sin embargo, un meta-análisis (Dantas et al. 2011, 2010; Oliveira et al. 2005; Sequeiros et al. 2005) han descrito el alcance del modelo tradicional de Matveyev (1977), como el más apropiado; no obstante, se esperan que próximas revisiones sistemáticas corroboren lo planteado frente al modelo tradicional (Dantas et al. 2011), ya que tal y como coincide con los resultados obtenidos, los modelos emergentes como el ATR y las Campanas Estructurales, se están mostrando con un índice de aplicabilidad de "Muy Bueno" (Dantas et al. 2010).

Por grupos de deportes, los de resistencia, priorizan los mesociclos especiales, mientras los de fuerza-velocidad, se inclinaron por los de base y competitivos. En deportes de pelota, el énfasis fue sobre los de control y especiales; en los de combate, los precompetitivos y competitivos y en los PAC, los de control.

Según Issurin (2012), los tipos de microciclos son de Adaptación, que duran de tres a cinco días y se caracterizan por un aumento gradual de la carga, que oscilan entre 5 y 9 días, utilizando cargas grandes y medias, para desarrollar la condición física; los de Impacto o choque, de 4 a 7 días, con cargas extremas; los de Precompetencia, de 5 a 7 días, que buscan la puesta a punto; los de Competencia, de 2 a 7 días y los de Recuperación Activa, de 3 a 7 días, empleando un amplio espectro de medios de recuperación. Los entrenadores suramericanos concuerdan con los mismos, con pequeñas diferencias en su denominación, que no afecta lo fundamental.

Al respecto, se encontró que existe una alta concordancia con los mesociclos, los cuales, se centran en los periodos competitivos, determinados por los microciclos de choque y competitivos, seguidos de los pre-competitivos. En los deportes de resistencia, los entrenadores hacen alternancia de la planificación entre la competencia, la alta exigencia deportiva y la recuperación o regeneración en el proceso de periodización y planificación. La fuerza y velocidad son capacidades que se determinan por características genéticas y de desarrollo continuo, por eso se puede entender que los entrenadores de deportes que tienen estas características centran el proceso en microciclos graduales, para acumular grandes volúmenes de carga, que les permita soportar luego microciclos de choque y competitivos.

Los deportes de pelota, por las características del calendario de competencias y dado que sus salidas de competencia son de mayor frecuencia que otros tipos de deportes, la planificación se centra en periodos competitivos, pero, esencialmente, en los micros competitivos, de choque y precompetitivos, alternados con los de regeneración. Los entrenadores de los deportes de combate se centran en lo competitivo (micros de choque, competitivo y precompetitivo), alternando los de regeneración. En condiciones similares a los deportes de combate, se analiza la planificación y la periodización de los deportes PAC, rescatando los micros de choque y precompetitivos, como plataforma a la competencia y su respectiva regeneración y recuperación en el proceso.

El contenido de los microciclos tiene una de dos tendencias: carga de efecto limitado, por ejemplo, orientada hacia una sola capacidad condicional o bien, entrenamiento combinado, en el cual, en cada microciclo se trabajan diferentes capacidades y componentes del rendimiento. La primera orientación es conocida como entrenamiento de bloques y 
constituye la última revolución marcada en la metodología del entrenamiento. Dicho método es utilizado por un 13,3\% de los entrenadores suramericanos, especialmente, en el grupo de deportes de fuerza-velocidad.

Duración de los planes de entrenamiento: En este contexto, se destaca una diferencia entre los diversos grupos de deportes, lo que señala que los entrenadores no utilizan una misma duración del plan de entrenamiento. Excepto en los deportes de resistencia, los planes a largo y mediano plazoson los más empleados, mientras en aquellos prevalecen tipos de planificación como olímpico y anual (Tabla 4).
Por otro lado, cabe resaltar la variedad de modelos de planes de entrenamiento en los deportes de pelota, donde se emplean todos los existentes, que puede estar dado por los mismos criterios de planificación de las competencias y de los objetivos, aspecto comentado anteriormente en el apartado de los microciclos competitivos y de recuperación. Se aprecia que los planes plurianuales y perspectivos sólo son empleados en deportes de pelota. El Plan Olímpico es el más empleado en el grupo de deportes de resistencia y en deportes de PAC.

Tabla 4. Duración de los planes, los períodos y las sesiones de entrenamiento utilizados por los entrenadores suramericanos, según grupos de deportes.

\begin{tabular}{|c|c|c|c|c|c|c|c|c|c|c|c|c|}
\hline \multirow[t]{2}{*}{ Variable } & \multicolumn{2}{|c|}{$\begin{array}{l}\text { Todos } \\
(\mathrm{n}=93)\end{array}$} & \multicolumn{2}{|c|}{$\begin{array}{l}\text { Resist. } \\
(\mathrm{n}=9)\end{array}$} & \multicolumn{2}{|c|}{$\begin{array}{l}\text { Fza-vel } \\
(n=16)\end{array}$} & \multicolumn{2}{|c|}{$\begin{array}{l}\text { Pelota } \\
(\mathrm{n}=37)\end{array}$} & \multicolumn{2}{|c|}{$\begin{array}{c}\text { Combate } \\
(n=15)\end{array}$} & \multicolumn{2}{|c|}{$\begin{array}{c}\text { PAC } \\
(n=16)\end{array}$} \\
\hline & $\mathrm{n}$ & $\%$ & $\mathrm{n}$ & $\%$ & $\mathrm{n}$ & $\%$ & $\mathrm{n}$ & $\%$ & $\mathrm{n}$ & $\%$ & $\mathrm{n}$ & $\%$ \\
\hline \multicolumn{13}{|c|}{ Tipo de planificación empleada en función de su duración } \\
\hline Largo Plazo & 38 & 25,9 & 2 & 15,4 & 9 & 29,0 & 15 & 25,4 & 6 & 31,6 & 6 & 28,6 \\
\hline MedianoPlaz & 28 & 19,0 & 1 & 7,7 & 7 & 22,6 & 12 & 20,3 & 4 & 21,1 & 4 & 19,0 \\
\hline Olímpico & 26 & 17,7 & 4 & 30,8 & 5 & 16,1 & 8 & 13,6 & 4 & 21,1 & 5 & 23,8 \\
\hline Anual & 24 & 16,3 & 4 & 30,8 & 4 & 12,9 & 8 & 13,6 & 4 & 21,1 & 3 & 14,3 \\
\hline CortoPlazo & 21 & 14,3 & 2 & 15,4 & 4 & 12,9 & 12 & 20,3 & 1 & 5,3 & 2 & 9,5 \\
\hline Plurianual & 6 & 4,1 & 0 & 0,0 & 0 & 0,0 & 2 & 3,4 & 0 & 0,0 & 1 & 4,8 \\
\hline Perspectivo & 4 & 2,7 & 0 & 0,0 & 2 & 6,5 & 2 & 3,4 & 0 & 0,0 & 0 & 0,0 \\
\hline \multicolumn{13}{|c|}{ Duración de los períodos (meses) } \\
\hline & $\min$ & $\max$ & $\min$ & $\max$ & $\min$ & $\max$ & $\min$ & $\max$ & $\min$ & $\max$ & $\min$ & $\max$ \\
\hline Preparat & 4,1 & 1,9 & 4,1 & 1,2 & 4,6 & 2,1 & 3,5 & 2,0 & 5,3 & 1,8 & 3,8 & 1,7 \\
\hline Competitivo & 2,8 & 1,7 & 3,6 & 2,8 & 2,1 & 1,1 & 2,7 & 1,5 & 3,0 & 1,7 & 3,1 & 2,2 \\
\hline Transitorio & 1,5 & 1,2 & 1,5 & 1,4 & 1,1 & 0,3 & 2,0 & 1,6 & 1,0 & 0,0 & 1,4 & 0,5 \\
\hline \multicolumn{13}{|c|}{ Número de sesiones semanales por período } \\
\hline & $\min$ & $\max$ & $\min$ & $\max$ & $\min$ & $\max$ & $\min$ & $\max$ & $\min$ & $\max$ & $\min$ & $\max$ \\
\hline Preparat & 1 & 18 & 6,6 & 8,2 & 6,6 & 11,3 & 5,9 & 9,2 & 7,7 & 11,4 & 5,1 & 8,1 \\
\hline Competitivo & 2 & 27 & 6,6 & 9,3 & 6,1 & 7,3 & 4,5 & 7,1 & 7,7 & 9,4 & 4,5 & 6,6 \\
\hline Transitorio & 1 & 12 & 4,0 & 7,0 & 4,7 & 7,8 & 3,2 & 4,7 & 4,4 & 6,0 & 2,8 & 5,1 \\
\hline \multicolumn{13}{|c|}{ Duración de las sesiones (horas) } \\
\hline & $\min$ & $\max$ & $\min$ & $\max$ & $\min$ & $\max$ & $\min$ & $\max$ & $\min$ & $\max$ & $\min$ & $\max$ \\
\hline Preparat. & 1,9 & 3,0 & 1,9 & 3,0 & 1,4 & 2,4 & 1,8 & 2,8 & 1,7 & 2,5 & 2,9 & 4,7 \\
\hline Competitivo & 1,6 & 2,6 & 1,9 & 2,7 & 1,4 & 2,0 & 1,4 & 2,2 & 1,6 & 2,8 & 2,2 & 4,7 \\
\hline Transitorio & 1,5 & 2,2 & 1,4 & 1,9 & 2,2 & 2,5 & 1,2 & 1,9 & 1,0 & 1,8 & 1,9 & 2,8 \\
\hline
\end{tabular}

Nota: Resist=deportes de resistencia, Fza-vel=deportes de fuerza-velocidad, PAC=deportes de Precisión y arte competitivo, Precomp $=$ precompetitivo, competi $=$ competitivo, regener $=$ regenerativo. 
En relación a la duración de los periodos de entrenamiento, se encontró que los deportes de combate, seguidos de los de fuerza-velocidad, son los que más tiempo emplean en el periodo preparatorio, a la vez, que se constituyen en los que menos tiempo dedican al periodo transitorio, lo que es totalmente distinto a los deportes de pelota, quienes menos tiempo dedican a su preparación y son quienes más amplio tienen el periodo transitorio. Los deportes de resistencia son en los que más tiempo dura el periodo de competencias.

Acerca de la cantidad de sesiones semanales por periodo, los deportes de fuerza-velocidad y combate son los que mayor cantidad presentaron. Se podría señalar que los deportes de pelota que son los que menos tiempo dedicaban en su periodo de preparación, en esta misma lógica tendrían también el menor número de sesiones semanales; sin embargo, son desplazados a una segunda ubicación por el grupo de PAC. También estos deportes son los que menos dedican al periodo transitorio, en tanto que los deportes con mayor cantidad de sesiones en el período transitorio son los de fuerza-velocidad. En esencia, se destaca que los deportes de fuerza-velocidad son aquellos que dedican más sesiones durante los tres periodos de planificación.

Frente a la variable de la cantidad de horas de las sesiones, sobresalen los deportes de precisión y arte competitivo, los cuales, en los periodos de preparación y de competencia ocupan una cantidad elevada en comparación a otros deportes y se puede explicar, debido a la cantidad de repeticiones que requieren ejecutar en función del perfeccionamiento del gesto técnico. Durante el periodo de competencia quienes menos tiempo ocupan son los deportes de fuerza-velocidad.

Según Navarro et al. (2010), las sesiones son de tres tipos: selectivas, complejas y suplementarias, caracterizadas por una alta concentración de un contenido del entrenamiento (todos los ejercicios se dirigen hacia un componente de la preparación), o por la posibilidad de mejorar diferentes capacidades y elevar la carga total de trabajo o por estar dirigidas a un objetivo auxiliar y a suplementar el programa de entrenamiento básico, respectivamente. Las sesiones complejas suelen ser más largas, a fin de poder cumplir con los propósitos expuestos. En este sentido, la duración de las sesiones reportada varió entre 1,5 y 3 horas, permitiendo asumir que emplearon los diferentes tipos de sesiones.

Los entrenadores describieron que el tiempo sobre el que hacen sus planificaciones, en primer lugar, es el largo plazo, seguido del mediano, duraciones que son una característica del deporte actual, con hasta 15 y 20 años, que van desde los inicios de la formación del niño deportista hasta la longevidad deportiva, pasando por la fase de los máximos resultados. Se encontró una diferencia entre los mejores cla- sificados y los siguientes, puesto que mientras los primeros prefieren la planificación a largo plazo, los últimos, en la tabla de medallas, lo hacen a mediano y corto plazo. Los ciclos olímpicos también son preferidos entre los mejores países por resultados.

Algunos entrenadores manifestaron que no estaban vinculados laboralmente o que solo trabajaban ad-honorem con sus deportistas, o que la vinculación no tenía la permanencia necesaria para garantizar la continuidad de los procesos de preparación de deportistas hacia el alto rendimiento, lo cual, hacía que algunos elaboraran sus planes solo cuando tenían asegurada su participación en eventos cercanos o la vinculación laboral. Esto, por lógica, limita y afecta considerablemente los procesos de planificación y de periodización a largo plazo y, en especial, en el nivel al que se pueden proyectar los deportistas suramericanos.

En conclusión, se encontró una clara relación entre la formación académica de los entrenadores y la clasificación general final de los Juegos (por medallas de oro).En cuanto a los modelos de planificación más utilizados predominaron el modelo tradicional, los ciclos, los bloques e individual y el ATR. El calendario de competencias es el criterio que más se tiene en cuenta para la planificación del entrenamiento deportivo, seguido de cerca por los objetivos y las características del deportista.

Los entrenadores suramericanos priorizaron los mesociclos competitivos y especiales, seguidos de cerca por los de control. Los deportes de resistencia, priorizaron los mesociclos especiales, mientras los de fuerza-velocidad, se inclinaron por los de base y competitivos. En deportes de pelota, el énfasis fue sobre los de control y especiales, en los deportes de combate, los precompetitivos y competitivos y en los PAC, los de control.

En cuanto a la duración de los planes de entrenamiento, se encontró una alta frecuencia de entrenadores (en deportes de fuerza-velocidad, de pelota, de combate y PAC), que indican que los planes a largo y mediano plazo son los más empleados. En cambio, en los deportes de resistencia prevalecieron los tipos de planificación olímpico y anual.

Frente a la cantidad de horas de las sesiones, sobresalen los deportes de precisión y arte competitivo, los cuales, en los periodos de preparación y de competencia ocupan una cantidad elevada en comparación a otros deportes, que se puede explicar por la cantidad de repeticiones que requieren ejecutar en función del perfeccionamiento del gesto técnico. Durante el periodo de competencia quienes menos tiempo ocupan son los deportes de fuerza-velocidad. 
Agradecimientos: Al Dr. Julio Roberto Gómez Gaitán, Gerente de INDEPORTES Antioquia. A la Vicerrectoría de Investigaciones y Postgrados de la Universidad de Caldas. Al licenciado Harold León y a los estudiantes del programa de Educación Física: René Quintero, William Cárdenas, William Dann y Camilo Saldarriaga, en la recolección de información. Conflicto de intereses: El manuscrito fue preparado y revisado con la participación de todos los autores, quienes declaramos que no existe ningún conflicto de intereses que ponga en riesgo la validez de los resultados esperados.

\section{BIBLIOGRAFÍA}

1. ACEVEDO, M. 2010. Técnicas Deportivas y su aplicación en las diferentes etapas de la vida del deportista. Ed. Gobernación de Nariño. (Colombia). 160p.

2. BOMPA, T. 2003. Teoría y metodología del entrenamiento. Ed. Hispano-Europea. (España). 429p.

3. DANTAS, E.H.; DE GODOY, E.S.; SPOSITO-ARAUJO, C.A.; OLIVEIRA, A.L; BESSA, A. 2011. Adequabilidade Dos Principais Modelos De Periodização Do Treinamento Esportivo. Rev. Bras. Ciênc. Esporte, (Brasil) 33(2):483-494.

4. DANTAS, E.; GARCÍA-MANSO, J.M.; GODOY, E.S.; SPOSITO-ARAUJO C.A.; GOMEZ, A.C. 2010. Aplicabilidad de los modelos de periodización del entrenamiento deportivo: una revisión sistemática. Intl. J. Sport Sci. 6(20):231-41.

5. GONZÁLEZ RAVE, J.M.; NAVARRO VALDIVIESO, F.J.; PEREIRA GASPAR, P.M. 2007. La planificación del entrenamiento deportivo: cambios vinculados a las nuevas formas de entender las estructuras deportivas contemporáneas. Conexões, Rev. Facultade Educação Física UNICAMP, Campinhas. 5(1):1-22.

6. HARRE, D. 1983. Teoría del entrenamiento deportivo. Ed. Científico-técnica. (Cuba). 394p.

7. HERNÁNDEZ-SAMPIERI, R.; FERNÁNDEZ, C.; BAPTISTA, M.P. 2010. Metodología de la Investigación. $5^{\mathrm{a}}$ ed. Ed. McGraw-Hill. (México). 613p.

8. ISSURIN, V.B. 2010.New horizons for the methodology and physiology of training periodization. Sports Med. (Estado Unidos) 40(3):189-207.

9. ISSURIN, V.B. 2012. Entrenamiento deportivo. Periodización enbloques. Barcelona: Paidotribo. (España). 417p.
10. MANZANO, J.I. 2004. Clarificación de conceptos relacionados con el entrenamiento deportivo. Rev. Escuela Abierta. (España) 4:55-71.

11. MATVEYEV, L.P. 1977. Bases del entrenamiento deportivo. Ed. Cultura Física y Deporte. (Rusia). 280p.

12. MATVEYEV, L.P. 2001. Teoría general del entrenamiento deportivo. Barcelona, Ed. Paidotribo. 268p.

13. NAVARRO, F. 2003. La Estructura Convencional de Planificación del Entrenamiento versus la Estructura Contemporánea. Disponible desde Internet en: http:// www.g-se.com/pid/229/ (con acceso 17/02/11).

14. NAVARRO, F.; OCA, A.; RIVAS, A. 2010. Planificación del entrenamiento y su control. Real Federación Española de Natación. Ed. Cultiva Libros. (España). 347p.

15. OLIVEIRA, A.L.; SEQUEIROS, J.L.S.; DANTAS, E.H.M. 2005 Comparative study between Matveev's classic periodization model and Verkhoshanski's blocks periodization model. (España). Fitness \& Performance J. 4(6):358-362.

16. PLATONOV, V. 2001. Teoría General del Entrenamiento Deportivo Olímpico. Ed. Paidotribo. (España). 684p.

17. SALINERO, J.J.; RUIZ, G. 2010. El entrenador de alto rendimiento deportivo y su contraste con entrenadores de menor nivel. Estudio en la modalidad de karate en España. Mot. Eur. J. Hum. Mov. (España) 24(1):107-119.

18. SEQUEIROS, J.L.S.; OLIVEIRA, A.L.B.; CASTANHEDE, D.; DANTAS, E.H.M. 2005. Estudio sobre la fundamentación del modelo de periodización de Tudor Bompa del entrenamiento deportivo. Fitness \& Performance J. 4(6):340-346.

19. VASCONCELOS, A. 2005. Planificación y organización del entrenamiento deportivo. Ed.Paidotribo. (España). 198p.

20. WEINECK, J. 2005. Entrenamiento total.Ed. Paidotribo. (España). 686p.

Recibido: Marzo 5 de 2012

Aceptado: Mayo 4 de 2012 